\title{
Limit cycle bifurcations in resonant LC power inverters under zero current switching strategy
}

\author{
Luis Benadero · Enrique Ponce · Abdelali El Aroudi · Francisco Torres
}

Received: date / Accepted: date

\begin{abstract}
The dynamics of a DC-AC self-oscillating LC resonant inverter with a zero current switching strategy is considered in this paper. A model that includes both the series and the parallel topologies and accounts for parasitic resistances in the energy storage components is used. It is found that only two reduced parameters are needed to unfold the bifurcation set of this extended system: one is related to the quality factor of the LC resonant tank and the other accounts for the balance between serial and parallel losses. Through a rigorous mathematical study, a complete description of the bifurcation set is obtained and the parameter regions where the inverter can work properly is emphasized.
\end{abstract}

Keywords Piecewise linear dynamics - Limit cycles · Bifurcations $\cdot$ Power inverters $\cdot$ ZCS control

E. Ponce and F. Torres are partially supported by the Spanish Ministerio de Economía y Competitividad, in the frame of project MTM201565608-P and by the Consejería de Economía y Conocimiento de la Junta de Andalucía under grant P12-FQM-1658. L. Benadero and A. El Aroudi were supported by the Spanish Ministerio de Ciencia e Innovación under grant DPI2013-47293-R.

\section{Benadero}

Departament de Física

Universitat Politècnica de Catalunya, Barcelona, Spain

E-mail: luis@fa.upc.edu

E. Ponce

Departamento de Matemática Aplicada

Escuela Técnica Superior de Ingeniería, Sevilla, Spain

E-mail: eponcem@us.es

\section{A. El Aroudi}

GAEI Research group, Departament d'Enginyeria Electrònica, Elèctrica i Automàtica

Universitat Rovira i Virgili, Tarragona, Spain

E-mail: abdelali.elaroudi@urv.cat

F. Torres

Departamento de Matemática Aplicada

Escuela Técnica Superior de Ingeniería, Sevilla, Spain

E-mail: ftorres@us.es

\section{Introduction}

Resonant inverters were introduced many years ago although their use has been confined for a long time to very specific applications, such as in high-voltage power supplies or audio amplifiers [1-3]. With the introduction of new regulations concerning efficiency and power density, power electronics designers have been pushed to find increasingly more efficient conversion systems. As a consequence, a renewed interest has emerged in resonant conversion. In particular, resonant inverters have recently gained more popularity in emerging applications such as wireless power transfer [4-6], battery charging in electrical vehicles [7], induction heating [8] and powering high intensity discharge lamps [9], among others.

Roughly speaking, resonant inverters are systems that include a switching network and a resonant LC tank circuit that converts a DC voltage into an AC voltage, thus providing AC power to a load. To do so, the switching network typically generates a square-wave signal that is applied to a resonant tank circuit. The switching frequency in resonant converters is usually very close to the resonant tank frequency and therefore the AC amplitude of the state variables is larger than the ripple of PWM hard switching converters. Furthermore, by rectifying and filtering the AC output of a resonant inverter, resonant DC-DC converters can be formed.

Different types of resonant inverters exist and these are classified according to the type of switching network, the configuration of the resonant tank circuit, and the number of the energy storage components. The parallel resonant converter (PRC) and the series resonant converter (SRC) are examples of topologies with two energy-storage elements $[10,11]$, corresponding to the simplest possible configurations. 
A major advantage of resonant inverters is their ability to operate with zero voltage switching (ZVS) or with zero current switching (ZCS) [12]. These techniques help to minimize switching losses even when inverters work at high switching frequency, hence allowing the use of small storage components. Therefore, the power density is improved. Another benefit is the low electromagnetic interference that appears in resonant topologies.

Regarding the physical realization of resonant inverters, a serious problem for designers comes from the lack of a rigorous analysis accounting for the dynamical behavior and the stability of oscillations. That is why in the majority of the reported works, the results are not general and can only be applied to particular cases. The purpose of this paper is to provide a sound approach to deal with this problem and to get a deep insight into the dynamics of these systems.

A rather general piecewise linear (PWL) model including parasitic resistances for energy storage elements considering both parallel and series design is introduced in this paper. We assume a ZCS control and study thoroughly the dynamics that can be obtained depending on the choice of different parameters. For that, we derive a canonical model with only two parameters: one of them is related to the quality factor of the LC circuit while the other accounts for the balance between serial and parallel losses. The two extreme values of this second parameter address to the ideal series and the parallel cases. The bifurcation set is obtained through the rigorous mathematical description of the different bifurcation curves. The parameter region where the inverter should work for a proper operation is emphasized.

The study of PWL systems can be a difficult task that is not within the scope of traditional analysis techniques for nonlinear systems. In particular, a sound bifurcation theory is lacking for such systems due to their nonsmooth character, and so their study must be done case by case e.g., $[13,14]$. Enforced by modern nonlinear engineering problems, some works in the literature on planar PWL systems deal with vector fields where continuity at the boundary is not assumed.

Dealing with non-smooth systems, after the pioneering work of Filippov [15], many efforts have been devoted to their bifurcation and stability analysis. One-parameter bifurcations for these systems are throughly studied in [16]. In particular, some specific cases are considered in [17-19]. In [20], it was shown that when there exists a generalized singular point of pseudo-focus type, degenerate Hopf bifurcations are possible. Later, other studies analyzing perturbations of systems in the so-called focus-focus (FF) case appeared. For instance, the possible existence of two small limit cycles under general perturbations of FF systems that can move the equilibrium points out of the boundary is reported in [21]. The number of bifurcating limit cycles increases when the two involved subsystems share the linear part of focus type and different quadratic terms are consid-

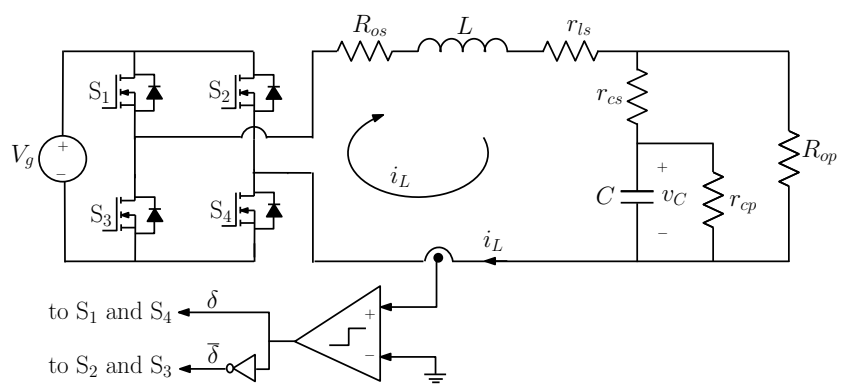

Fig. 1 Generalized schematic diagram of an LC resonant inverter. Here, some extra elements have been included to account for both parallel and series resonant converters ans also for parasitic elements. Note that if a resistor is missing, either its corresponding conductance or resistance vanishes.

ered, see [22], or even more when the linear terms do not coincide, see for instance [23] and the references therein.

Restricting our attention to discontinuous PWL systems with two zones separated by a straight line, some works have been done. In particular, in [24] a similar situation to the one appearing in our model is considered, namely systems with a global vector field symmetrical with respect to the origin.

In this paper, a planar discontinuous PWL dynamical system with symmetry and two linear zones is obtained to model a self-oscillating LC resonant inverter. At the discontinuity line a repulsive sliding set exists that plays a key role in some limit cycle bifurcations. It is shown that the boundaries of attraction of the existing equilibrium points are determined by unstable closed loops. The bifurcation pattern, which includes a saddle-node bifurcation of limit cycles, is extensively analyzed, getting a valuable information for the correct operation and design of the inverter.

The rest of the paper is organized as follows. In Section 2 , the mathematical model of the proposed general inverter is obtained and some canonical forms are derived in order to minimize the number of parameters for its analysis. Section 3 is devoted to the complete determination of the bifurcation set regarding the two essential parameters previously introduced. In Section 4, some qualitative features regarding oscillations are stressed and the safety region of the circuit is highlighted. Finally, some concluding remarks are summarized in the last section.

\section{System description and mathematical modeling}

\subsection{System Description}

Figure 1 shows a generalized circuit diagram of a resonant DC-AC H-bridge inverter. In this diagram, some extra elements have been included in such a way that both parallel and series resonant converters can be studied. Also, parasitic resistances in the energy storage elements are taken into account. In that diagram, the output series resistance $R_{o s}$; the 
inductor with inductance $L$ and parasitic series resistor $r_{l s}$; the output parallel conductance $G_{o p}=1 / R_{o p}$; and the capacitor with capacitance $C$ with parasitic parallel conductance $g_{c p}=1 / r_{c p}$ and parasitic series resistance $r_{c s}$ can be identified. Other elements in the circuit diagram in Fig. 1 are the input source voltage $V_{g}$, and the switches $\mathrm{S}_{1}, \mathrm{~S}_{2}, \mathrm{~S}_{3}$ and $\mathrm{S}_{4}$.

The circuit operation is based on an automatically activated switching between two configurations driven by the signals $\delta$ and $\bar{\delta}=1-\delta$. The switches $\mathrm{S}_{1}$ and $\mathrm{S}_{4}$ are $\mathrm{ON}$ when $i_{L}>0$ (therefore $\delta=1$ ), and they are turned OFF when $i_{L}<0(\delta=0)$, where $i_{L}$ is the current through the inductor $L$. The switches $S_{2}$ and $S_{3}$ are respectively driven in a complementary way to $S_{1}$ and $S_{4}$, so that a ZCS control is implemented.

It is clear that without switching, the capacitor voltage and the inductor current tend to some constant values. In other words, when $\delta=1$ there is always an equilibrium point with positive values for the capacitor voltage and the inductor current and when $\delta=0$ there is another equilibrium point with negative values for those magnitudes.

The goal of the switching control is to take advantage of the transient regimes associated to these equilibria. Then, by building a suitable aggregation of orbits, a limit cycle, which is the desired oscillating behavior in steady state regime, is made up. This goal will be possible only under certain restrictions, as shown later. Even when the switching is acting, the dynamics could be addressed to one of these equilibrium points depending on the starting conditions, which is undesirable. For instance, if the quality factor of the LC circuit is lower than a critical value to be determined later, the normal zero initial condition lies on the attraction basin of the undesired equilibria and therefore the limit cycle would not be reached. On the contrary, an appropriate choice of parameters will warrant a good performance for the inverter under standard initial conditions. Such a choice of parameters only can be soundly made after an analysis of all the possible dynamics in the circuit. As an important feature to be fulfilled, the attraction basin of these equilibria should be minimized as much as possible to get a robust operation for the inverter.

\subsection{State-space model}

Let $v_{C}$ be the voltage of the capacitor $C$ and recalling $i_{L}$ is the current in the inductor $L$, then, by applying Kirchoff voltage and current laws, we get

$i_{L}=i_{c s}+\left(i_{c s} r_{c s}+v_{C}\right) G_{o p}$

$u V_{g}=L \frac{\mathrm{d} i_{L}}{\mathrm{~d} t}+i_{L}\left(R_{O S}+r_{l s}\right)+i_{c s} r_{c S}+v_{C}$,

where $i_{c s}$, that is the current through $r_{c s}$, is

$i_{c s}=C \frac{\mathrm{d} v_{C}}{\mathrm{~d} t}+g_{c p} v_{C}$.
After some algebra, the following model is obtained

$\frac{\mathrm{d}}{\mathrm{d} t}\left(\begin{array}{c}v_{C} \\ i_{L}\end{array}\right)=\mathbf{A}\left(\begin{array}{c}v_{C} \\ i_{L}\end{array}\right)+u \mathbf{b}$

where

$\mathbf{A}=\left(\begin{array}{cc}-\frac{G_{p}}{C} & \bar{\kappa} \\ -\frac{\kappa}{L} & -\frac{R_{s}}{L}\end{array}\right), \quad \mathbf{b}=\left(\begin{array}{c}0 \\ \frac{V_{g}}{L}\end{array}\right)$,

and the factor $\kappa$ is introduced to define the equivalent series resistance $R_{s}$ and the equivalent parallel conductance $G_{p}$,

$\kappa=\frac{1}{1+r_{c s} G_{o p}}$

$R_{s}=R_{o s}+r_{l s}+\kappa r_{c s}, \quad G_{p}=g_{c p}+\kappa G_{o p}$.

The variable $u=2 \delta-1$ is determined by the control, such that $u=1$ (that is, $\delta=1$ ) if $i_{L}>0$, and $u=-1$ (that is, $\delta=0$ ) if $i_{L}<0$. This control strategy based on the use of the sign of the inductor current in the switching decision is called zero current switching (ZCS). Then, the switching condition, which is in general a function of the state variables and time, depends only on the inductor current and it can be expressed in terms of the state variables as

$h\left(v_{C}, i_{L}\right)=i_{L}$,

so that the ZCS control strategy leads to $u=\operatorname{sign}\left(h\left(v_{C}, i_{L}\right)\right)$ and the system is autonomous.

Next, in order to simplify the analysis of its possible dynamics and bifurcations, system (1)-(2) will be normalized into a simpler form with a minimum number of parameters.

\subsection{Canonical forms}

In this section, we will obtain the Frobenius or rational canonical form for the common linear part, which is well suited for our subsequent analysis. Doing so, the needed changes of variables preserve the discontinuity line, so that the second variable is still associated to the electrical current across the inductance. Note that in general, if we choose instead the Jordan canonical form, then the discontinuity line should not coincide with any of the coordinate axes.

First, we consider the natural frequency $\omega_{0}$ and the quality factor $Q$ of the two linear subsystems in (1),

$$
\begin{aligned}
& \omega_{0}=\sqrt{\operatorname{det}(\mathbf{A})}=\sqrt{\frac{R_{s} G_{p}+\kappa^{2}}{L C}}, \\
& \frac{1}{Q}=-\frac{\operatorname{tr}(\mathbf{A})}{\omega_{0}}=\frac{G_{p}}{\omega_{0} C}+\frac{R_{s}}{\omega_{0} L},
\end{aligned}
$$


where $\operatorname{det}(\mathbf{A})$ and $\operatorname{tr}(\mathbf{A})$ stand for the determinant and the trace of matrix $\mathbf{A}$, respectively. Also, we introduce a parameter $\beta$ defined as

$\beta=\frac{Q G_{p}}{\omega_{0} C}=1-\frac{R_{s} Q}{\omega_{0} L}=\frac{G_{p} L}{G_{p} L+C R_{s}}$,

where all the equivalent expressions for $\beta$ come from (3).

It is worth noting that $0 \leq \beta \leq 1$. The case $\beta=0$ arises when $G_{p}=0$, and it corresponds to the ideal series inverter, i.e., the resistors $G_{o p}$ and $r_{c p}$ are absent. The case $\beta=1$ arises when $R_{s}=0$, i.e., the resistors $R_{o s}, r_{l s}$ and $r_{c s}$ are absent and it corresponds to the ideal parallel inverter.

In searching for a canonical form, the state vector is redefined by using the change of variables

$\mathbf{x}=\left(\begin{array}{l}x_{1} \\ x_{2}\end{array}\right)=\left(\begin{array}{cc}\frac{\kappa}{V_{g}} & -\frac{L G_{p}}{C V_{g}} \\ 0 & \frac{\omega_{0} L}{V_{g}}\end{array}\right)\left(\begin{array}{c}v_{C} \\ i_{L}\end{array}\right)$,

and the independent variable is changed by using a new time $\tau=\omega_{0} t$ so that the following proposition is obtained.

Proposition 1 System (1)-(2) can be reduced to

$\frac{\mathrm{d} \mathbf{x}}{\mathrm{d} \tau}=\mathbf{A x}+u \mathbf{b}$,

$h(\mathbf{x})=x_{2}$,

where matrix $\mathbf{A}$ and vector $\mathbf{b}$ are redefined as

$\mathbf{A}=\left(\begin{array}{cc}0 & 1 \\ -1 & \frac{-1}{Q}\end{array}\right), \quad \mathbf{b}=\left(\begin{array}{c}-\frac{\beta}{Q} \\ 1\end{array}\right)$

and $u=1$ if $h(\mathbf{x})>0$ or $u=-1$ if $h(\mathbf{x})<0$.

According to (6), the switching manifold $\Sigma$ is defined as $\Sigma=\left\{\left(x_{1}, x_{2}\right): x_{2}=0\right\}$,

and so the state space of the canonical form has two linearity regions, namely

$\Sigma^{+}=\left\{\left(x_{1}, x_{2}\right): x_{2}>0\right\}, \quad \Sigma^{-}=\left\{\left(x_{1}, x_{2}\right): x_{2}<0\right\}$.

The system (5), with a constant switch variable $u$, has the two equilibria

$\overline{\mathbf{x}}^{ \pm}= \pm\left(\bar{x}_{1}, \bar{x}_{2}\right)= \pm\left(1-\frac{\beta}{Q^{2}}, \frac{\beta}{Q}\right)$,

where it is assigned $\overline{\mathbf{x}}^{+}$and $\overline{\mathbf{x}}^{-}$to $u=1$ and $u=-1$ respectively. Whenever $\beta>0$, since $\bar{x}_{2}>0$, we deduce that $\overline{\mathbf{x}}^{+} \in \Sigma^{+}$and $\overline{\mathbf{x}}^{-} \in \Sigma^{-}$, so both equilibria are real. However, if $\beta=0$ (the ideal series inverter case), then the two equilibria are located at the points $\mathbf{x}^{ \pm}= \pm(1,0) \in \Sigma$, that is they lie at the switching line.
For these new variables the natural frequency is the unity and the poles $p^{ \pm}$of system (5), that is, the eigenvalues of matrix $\mathbf{A}$, are

$p^{ \pm}=-\frac{1}{2 Q} \pm \sqrt{\frac{1}{4 Q^{2}}-1}$.

Here, two different cases appear depending on the value of the quality factor $Q$. If $Q \leq 1 / 2$ then the eigenvalues are real and negative, so that equilibria are stable nodes. On the other hand, if $Q>1 / 2$ then both eigenvalues are complex with negative real part, that is, equilibria are stable foci. In the piecewise smooth system (5)-(6) with the parameter condition $\beta=0$, the node-focus transition at the value $Q=1 / 2$ gives rise to a non smooth bifurcation to be studied later.

When $Q \leq 1 / 2$ the equilibria have some invariant manifolds associated to the real eigenvalues, so that orbits cannot cut such invariant manifolds. As a consequence, any trajectory can cross the switching manifold $\Sigma$ at most once, precluding the oscillatory dynamics.

When $Q>1 / 2$, oscillatory dynamics can appear. The eigenvalues of matrix $\mathbf{A}$ given in (5) are now

$p^{ \pm}=-\frac{1}{2 Q} \pm i \sqrt{1-\frac{1}{4 Q^{2}}}=\sigma \pm i \nu$.

Note that these eigenvalues are located on the unit circle because $\sigma^{2}+v^{2}=1$. Then it turns out more convenient to study the dynamical behavior of our system by means of a new parameter $\gamma$ which is associated to the focus contraction when $\gamma<0$ or the focus expansion when $\gamma>0$. The parameter $\gamma$ will be crucial to study the bifurcations of system (1)-(2) and it is introduced as follows,

$\gamma=\frac{\sigma}{v}=\frac{-1}{\sqrt{4 Q^{2}-1}}<0$.

Notice that as $\gamma<0$, the focus is contractive. We are then in position of stating the following result.

Proposition 2 Assuming $Q>1 / 2$, system (1)-(2) can be reduced to

$\frac{\mathrm{d} \mathbf{x}}{\mathrm{d} \theta}=\mathbf{A} \mathbf{x}+u \mathbf{b}$,

$h(\mathbf{x})=x_{2}$

where matrix $\mathbf{A}$ and vector $\mathbf{b}$ are redefined as

$\mathbf{A}=\left(\begin{array}{cc}0 & 1+\gamma^{2} \\ -1 & 2 \gamma\end{array}\right), \quad \mathbf{b}=\left(\begin{array}{c}2 \beta \gamma \\ 1\end{array}\right)$

and $u=1$ if $h(\mathbf{x})>0$ or $u=-1$ if $h(\mathbf{x})<0$.

The system has two equilibria of focus type, with eigenvalues $\gamma \pm i$, namely

$\overline{\mathbf{x}}^{ \pm}= \pm\left(1-\frac{4 \beta \gamma^{2}}{1+\gamma^{2}}, \frac{-2 \beta \gamma}{1+\gamma^{2}}\right)$ 
Proof For any value of parameter $Q$, system (1)-(2) can be reduced to (5)-(6). If $Q>1 / 2$, the parameter $\gamma$ in (7) is well defined. Now, making in (5) the change of variables,

$\tilde{x}_{1}=x_{1}, \quad \tilde{x}_{2}=v x_{2}, \quad \theta=v \tau$

dropping tildes and taking into account that

$1+\gamma^{2}=\frac{1}{v^{2}}, \quad \frac{1}{v Q}=-2 \gamma$

system (8)-(9) is obtained. Equilibria computation is direct.

It is worth noting that if $\mathbf{x}(\boldsymbol{\theta})$ is a solution of system (8)-(9), then $-\mathbf{x}(\theta)$ is also a solution. As a consequence, an invariant closed curve $\Gamma$ is either symmetric with respect to the origin or there exists another invariant closed curve $\Gamma^{\prime}$ which is the symmetrical one to $\Gamma$ with respect to the origin.

It must be emphasized that the reduced systems (5)-(6) and (8)-(9) have only two parameters, $Q$ and $\beta$, or $\gamma$ and $\beta$ respectively. The parameters $Q$ and $\gamma$ are related to the global dissipation of the system while the parameter $\beta$ can be understood as the dissipation balance between series and parallel resistances.

In the following, we focus on the case $Q>1 / 2$, and according to Proposition 2, we study system (8)-(9).

\section{Sliding and crossing dynamics, limit sets and bifurcations}

First, the sliding dynamics is studied; afterwards, the crossing dynamics and possible periodic orbits will be addressed.

\subsection{Sliding set and pseudo equilibrium point}

In accordance to the defined switching manifold $\Sigma$ and the two corresponding state regions $\Sigma^{+}$and $\Sigma^{-}$, system (8)-(9) can be rewritten as $\dot{\mathbf{x}}=\mathbf{F}(\mathbf{x})$, where

$\mathbf{F}(\mathbf{x})=\left\{\begin{array}{l}\mathbf{F}^{+}(\mathbf{x})=\left(F_{1}^{+}(\mathbf{x}), F_{2}^{+}(\mathbf{x})\right)=\mathbf{A} \mathbf{x}+\mathbf{b}, \mathbf{x} \in \Sigma^{+}, \\ \mathbf{F}^{-}(\mathbf{x})=\left(F_{1}^{-}(\mathbf{x}), F_{2}^{-}(\mathbf{x})\right)=\mathbf{A} \mathbf{x}-\mathbf{b}, \mathbf{x} \in \Sigma^{-} .\end{array}\right.$

Orbits are well defined while they evolve without touching the switching manifold $\Sigma$. To define the orbits arriving at the discontinuity line $\Sigma$, we must distinguish between two different possibilities. If the two vector fields point to the same direction, we can concatenate solutions in a natural way. On the contrary, when such normal components are in opposition, we adopt the Filippov convex method. In the sequel, we will closely follow reference [16] to describe the Filippov method regarding system (11).
The product of the two normal components to the switching manifold, at a point $\mathbf{x}=\left(x_{1}, 0\right) \in \Sigma$ is

$\left(\nabla h(\mathbf{x}) \mathbf{F}^{+}(\mathbf{x})\right) \cdot\left(\nabla h(\mathbf{x}) \mathbf{F}^{-}(\mathbf{x})\right)=F_{2}^{+}(\mathbf{x}) F_{2}^{-}(\mathbf{x})=x_{1}^{2}-1$,

where $\nabla(\cdot)$ is the gradient operator, and then

$\nabla h(\mathbf{x})=(0,1), F_{2}^{+}(\mathbf{x})=-x_{1}+1, F_{2}^{-}(\mathbf{x})=-x_{1}-1$.

The crossing set $\Sigma^{c} \subset \Sigma$ is the set of all points $\mathbf{x} \in \Sigma$, where the normal components of $\mathbf{F}$ at both sides of the switching manifold have the same sign, that is

$\Sigma^{c}=\left\{\left(x_{1}, 0\right):\left|x_{1}\right|>1\right\}$.

At these points the orbits of system (11) cross the switching manifold $\Sigma$, i.e., orbits reaching $\Sigma$ from one zone concatenate in a natural way with orbits leaving $\Sigma$ and entering the other zone.

The sliding set $\Sigma^{s} \subset \Sigma$ is the complement in $\Sigma$ of the crossing set $\Sigma^{c}$, i.e.

$\Sigma^{s}=\left\{\left(x_{1}, 0\right):\left|x_{1}\right| \leq 1\right\}$

which is the set of all points $\mathbf{x} \in \Sigma$, where the normal components of the vector fields to the discontinuity line have opposite sign or one of them vanishes. Note that $F_{2}^{+}\left(x_{1}, 0\right)$ and $F_{2}^{-}\left(x_{1}, 0\right)$ do not simultaneously vanish at any point $\left(x_{1}, 0\right)$. The sliding set is repulsive because for system (11),

$F_{2}^{+}(\mathbf{x}) \geq 0$ and $F_{2}^{-}(\mathbf{x}) \leq 0, \quad \mathbf{x} \in \Sigma^{s}$.

The Filippov method associates to every $\mathbf{x} \in \Sigma^{s}$ the socalled sliding field $\mathbf{F}^{s}(\mathbf{x})$ by means of the convex combination

$\mathbf{F}^{s}(\mathbf{x})=\lambda \mathbf{F}^{-}(\mathbf{x})+(1-\lambda) \mathbf{F}^{+}(\mathbf{x})$,

where $\lambda=\lambda(\mathbf{x})$ is selected so that $\mathbf{F}^{s}(\mathbf{x})$ is tangent to the sliding set, that is

$\nabla h(\mathbf{x}) \mathbf{F}^{s}(\mathbf{x})=\lambda \nabla h(\mathbf{x}) \mathbf{F}^{-}(\mathbf{x})+(1-\lambda) \nabla h(\mathbf{x}) \mathbf{F}^{+}(\mathbf{x})=0$,

and then,

$\lambda(\mathbf{x})=\frac{\nabla \mathbf{h}(\mathbf{x}) \mathbf{F}^{+}(\mathbf{x})}{\nabla \mathbf{h}(\mathbf{x})\left(\mathbf{F}^{+}(\mathbf{x})-\mathbf{F}^{-}(\mathbf{x})\right)}$

For system (10), this implies

$\lambda\left(x_{1}\right)=\frac{F_{2}^{+}\left(x_{1}, 0\right)}{F_{2}^{+}\left(x_{1}, 0\right)-F_{2}^{-}\left(x_{1}, 0\right)}=\frac{1-x_{1}}{2}$.

Therefore, for $\left(x_{1}, 0\right) \in \Sigma^{s}$, the scalar differential equation

$\dot{x}_{1}=\lambda F_{1}^{-}\left(x_{1}, 0\right)+(1-\lambda) F_{1}^{+}\left(x_{1}, 0\right)=2 \beta \gamma x_{1}$

defines the so called sliding dynamics in the sliding set $\Sigma^{s}$ for system (8)-(9).

Solutions of (12) are called sliding solutions. In particular, constant sliding solutions are called pseudo-equilibria 
of the system. When $\beta=0$, equation (12) reduces to $\dot{x}_{1}=0$, and so every point in the sliding set is a pseudo-equilibrium point of system (11). When $\beta>0$, the origin is the only pseudo-equilibrium point. Taking into account that the sliding set $\Sigma^{s}$ is repulsive and that the origin is stable for the sliding dynamics, it turns out that system (11) has a pseudosaddle at the origin for $\beta>0$.

For system (11), the sliding set is delimited by the two points $\mathbf{x}_{B}^{ \pm}= \pm(1,0)$. If $\beta>0$, then $F_{2}^{+}\left(\mathbf{x}_{B}^{+}\right)=0$ and, recalling that $\gamma<0, F_{1}^{-}\left(\mathbf{x}_{B}^{+}\right)=2 \beta \gamma<0$. Moreover, since the orbit of the vector field $\mathbf{F}^{+}$passing through $\mathbf{x}_{B}^{+}$at a time, say $\theta=\theta_{B}$, belongs to $\Sigma^{+}$for $0<\left|\theta-\theta_{B}\right|<\varepsilon$, the point $\mathbf{x}_{B}^{+}$ is called a visible tangency point. Analogously, due to the symmetry of the vector field, the point $\mathbf{x}_{B}^{-}=-(1,0)$ is also a visible tangency point.

Although the system is discontinuous, for any initial condition $\mathbf{x}(0)$, it is possible to define, both in forward and backward time, a unique solution $\Phi(\theta, \mathbf{x}(0))$, with $\Phi(0, \mathbf{x}(0))=$ $\mathbf{x}(0)$. Of course, assuming $\mathbf{x}(0) \in \Sigma^{+}\left(\mathbf{x}(0) \in \Sigma^{-}\right.$is symmetrical), the corresponding solution $\Phi(\theta, \mathbf{x}(0))$ can be computed by solving $\dot{\mathbf{x}}=\mathbf{F}^{+}(\mathbf{x})$ while $\mathbf{x}(\theta) \in \Sigma^{+}$.

If a forward time solution $\mathbf{x}(\boldsymbol{\theta})$ does not reach the switching manifold $\Sigma$, it tends to the stable equilibrium $\mathbf{x}^{+}$. Otherwise, if the orbit reaches $\Sigma$ at the point $\left(x_{1}\left(\theta_{1}\right), 0\right)$ then necessarily $x_{1}\left(\theta_{1}\right) \geq 1$. If $x_{1}\left(\theta_{1}\right)>1$, then the orbit enters $\Sigma^{-}$and the vector field $\mathbf{F}^{-}$must be used to resume the computation. In the case $x_{1}\left(\theta_{1}\right)=1$, the system enters in sliding mode regime described by equation (12) and sliding takes place for $\theta>\theta_{1}$; if $\beta>0$, this orbit tends toward the pseudosaddle equilibrium at the origin and when $\beta=0$, then it is assumed that $\mathbf{x}(\theta)=\mathbf{x}\left(\theta_{1}\right)=(1,0)$ for all $\theta>\theta_{1}$.

In contrast, a backward time solution $\mathbf{x}(\theta)$ always reaches the discontinuity line $\Sigma$. Therefore, there exists a time $\theta_{2}<0$ for which $\mathbf{x}\left(\theta_{2}\right)=\left(x_{1}\left(\theta_{2}\right), 0\right) \in \Sigma$, where $x_{1}\left(\theta_{2}\right)<1$. At this point, the following possibilities arise when $\theta<\theta_{2}$.

(a) If $\beta \geq 0$ and $x_{1}\left(\theta_{2}\right)<-1$, the orbit crosses $\Sigma$ and $\mathbf{F}^{-}$ must be used to resume the reverse time computation.

(b) If $\beta=0$ and $-1 \leq x_{1}\left(\theta_{2}\right)<1$, then $\mathbf{x}(\theta)=\mathbf{x}\left(\theta_{2}\right)$ for all $\theta<\theta_{2}$.

(c) If $\beta>0$ and $-1 \leq x_{1}\left(\theta_{2}\right)<1$, then the following cases arise

(c1) If $x_{1}\left(\theta_{2}\right)=-1$, then the orbit crosses $\Sigma$ and then follows in backward time the unique standard orbit in $\Sigma^{-}$through the point $\mathbf{x}_{B}^{-}$.

(c2) If $-1<x_{1}\left(\theta_{2}\right)<0$, then for $\theta<\theta_{2}$, the orbit slides in backward time towards the point $\mathbf{x}_{B}^{-}$. Then, we proceed as in (c1).

(c3) If $x_{1}\left(\theta_{2}\right)=0$, then $\mathbf{x}(\theta)=\mathbf{x}\left(\theta_{2}\right)=(0,0)$ for all $\theta<\theta_{2}$.

(c4) If $0<x_{1}\left(\theta_{2}\right)<1$, then the orbit slides towards the point $\mathbf{x}_{B}^{+}$. Afterwards, the orbit follows in backward time the unique standard orbit in $\Sigma^{+}$through the point $\mathbf{x}_{B}^{+}$.
The different situations defined above are illustrated in Fig. 2. Observe that excepting the orbits tangent to the switching manifold $\Sigma^{s}$ at the points $( \pm 1,0)$, each panel can be obtained from the other one by reversing the sign of the arrows. Note also that system solutions are not invertible in the classical sense, since the orbits can overlap on the sliding set, see [16].

\subsection{Poincaré map associated to the switching manifold}

Taking into account the symmetry of the vector field with respect to the origin, we focus our attention on the upper half plane $\Sigma^{+}$, where the system has an equilibrium point $\left(\bar{x}_{1}, \bar{x}_{2}\right)$, see (10). Thus, by solving equation (8) with $u=1$, it turns out

$\left(\begin{array}{l}x_{1}(\theta)-\bar{x}_{1} \\ x_{2}(\theta)-\bar{x}_{2}\end{array}\right)=\phi(\theta)\left(\begin{array}{l}x_{1}(0)-\bar{x}_{1} \\ x_{2}(0)-\bar{x}_{2}\end{array}\right)$,

where $\phi(\theta)$ is the evolution operator given by

$\phi(\theta)=e^{\gamma \theta}\left(\begin{array}{cc}\cos \theta-\gamma \sin \theta & \left(1+\gamma^{2}\right) \sin \theta \\ -\sin \theta & \cos \theta+\gamma \sin \theta\end{array}\right)$.

We will resort to the auxiliary function

$\varphi_{\gamma}(\theta)=1-e^{\gamma \theta}(\cos \theta-\gamma \sin \theta)$

which was introduced in [13] and has the symmetry properties

$\varphi_{-\gamma}(\theta)=\varphi_{\gamma}(-\theta), \quad \varphi_{-\gamma}(-\theta)=\varphi_{\gamma}(\theta), \quad \gamma, \theta \in \mathbb{R}$.

Furthermore, the function $\varphi_{\gamma}$ has local maxima at $\theta=(2 n-$ 1) $\pi$ and local minima at $\theta=2 n \pi$, with $n \in \mathbb{Z}$. When $\gamma<0$, we denote as $\hat{\theta} \in(\pi, 2 \pi)$ the minimum positive value such that

$\varphi_{-\gamma}(\hat{\theta})=1-e^{-\gamma \hat{\theta}}(\cos \hat{\theta}+\gamma \sin \hat{\theta})=0$.

The function $\varphi_{-\gamma}(\theta)$ with $\gamma<0$ is represented in Fig. 3 .

Equation (15) implicitly defines a function $\hat{\theta}=\hat{\theta}(\gamma)$, whose first derivative is

$\frac{\mathrm{d} \hat{\theta}}{\mathrm{d} \gamma}=\frac{\sin \hat{\theta}-\hat{\theta}(\cos \hat{\theta}+\gamma \sin \hat{\theta})}{\left(\gamma^{2}+1\right) \sin \hat{\theta}}=\frac{\sin \hat{\theta}-\hat{\theta} e^{\gamma \hat{\theta}}}{\left(\gamma^{2}+1\right) \sin \hat{\theta}}>0$,

so the function $\hat{\theta}(\gamma)$ is increasing.

Taking now $\left(x_{1}(0), x_{2}(0)\right)=\left(z_{1}, 0\right)$, with $z_{1} \geq 1$ as initial point of an orbit and integrating backwards, the orbit evolves in the zone $\Sigma^{+}$surrounding the equilibrium point $\overline{\mathbf{x}}^{+}$until it eventually reaches the switching line $\Sigma$ at a point $(z, 0)$ with $z<1$. Thus, going in forward time a Poincaré map $P$ can be defined as $z_{1}=P(z)$.

The direction of the flow assures that orbits starting at the points $\left(z_{1}, 0\right)$ with $z_{1}>1$, go into the zone $\Sigma^{-}$, until they 


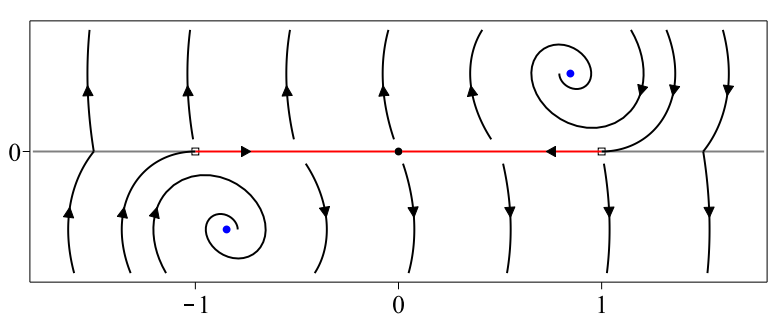

(a) Orbits in forward time.

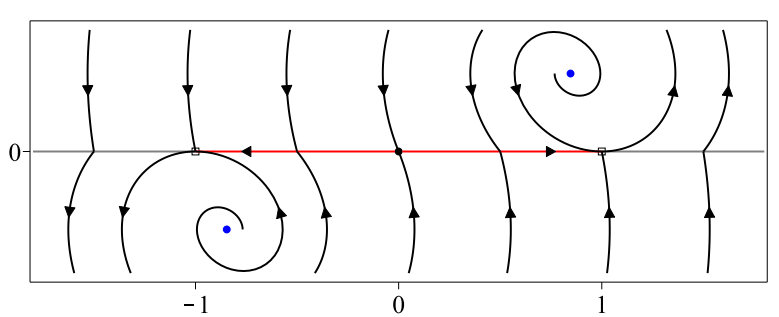

(b) Orbits in backward time.

Fig. 2 Orbits in a neighborhood of the sliding set when $\beta>0$. Left panel: The sliding set is repulsive and the only two orbits entering the sliding set are the orbits arriving tangentially to the two points $( \pm 1,0)$. Right panel: In backward time the sliding set is attractive and orbits arriving at points $( \pm x, 0)$ with $0<x \leq 1$ leave the sliding set through the points $( \pm 1,0)$.

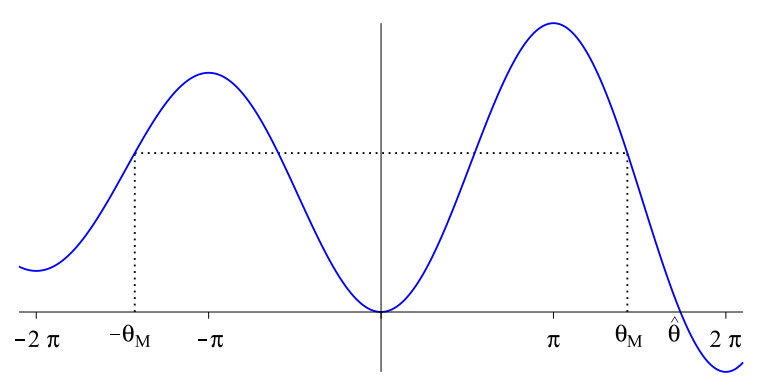

Fig. 3 Graph of function $\varphi_{-\gamma}(\theta)$ with $\gamma<0$. The value $\hat{\theta}$ is defined by $\varphi_{-\gamma}(\hat{\theta})=0$, with $\pi<\hat{\theta}<2 \pi$. The value $\theta_{M}$ is uniquely defined by $\varphi_{-\gamma}\left(-\theta_{M}\right)=\varphi_{-\gamma}\left(\theta_{M}\right)$ or equivalently $\varphi_{\gamma}\left(\theta_{M}\right)=\varphi_{\gamma}\left(-\theta_{M}\right)$, with $\pi<\theta_{M}<\hat{\theta}$.

reach $\Sigma$ again at a point $\left(z_{2}, 0\right)$ with $z_{2}<-1$. Therefore, we can define another Poincaré map $\widetilde{P}$ as $z_{2}=\widetilde{P}\left(z_{1}\right)$, for $z_{1}>1$.

It is direct to see that when $(\widetilde{P} \circ P)(z)=z$, a crossing limit cycle exists and so, the crossing limit cycles correspond to fixed points of the map $\widetilde{P} \circ P$.

Assuming that $P(z)=z_{1}$, we realize that the symmetry of the system imposes that $\widetilde{P}(-z)=-z_{1}$, and so

$(\widetilde{P} \circ P)(z)=-P(-P(z))=(-P) \circ(-P)(z)$.

Consequently, in looking for crossing limit cycles it is sufficient to determine the points $z<-1$ such that $P(z)=$ $-z$. Moreover, the derivative of the full Poincaré map $\widetilde{P} \circ P$ at a fixed point $z$ is

$(\widetilde{P} \circ P)^{\prime}(z)=-P^{\prime}(-P(z))\left(-P^{\prime}(z)\right)=\left(-P^{\prime}(z)\right)^{2}$,

hence, the fixed point is stable when $\left|P^{\prime}(z)\right|<1$ and unstable when $\left|P^{\prime}(z)\right|>1$.

Next the Poincaré map $P$ is determined. Two cases arises depending on the value of $\beta$.

\subsubsection{The case $\beta=0$}

When $\beta=0$, the equilibrium point of the vector field $\mathbf{F}^{+}$is located at $(1,0)$. Then, by solving equation (13), the Poincaré

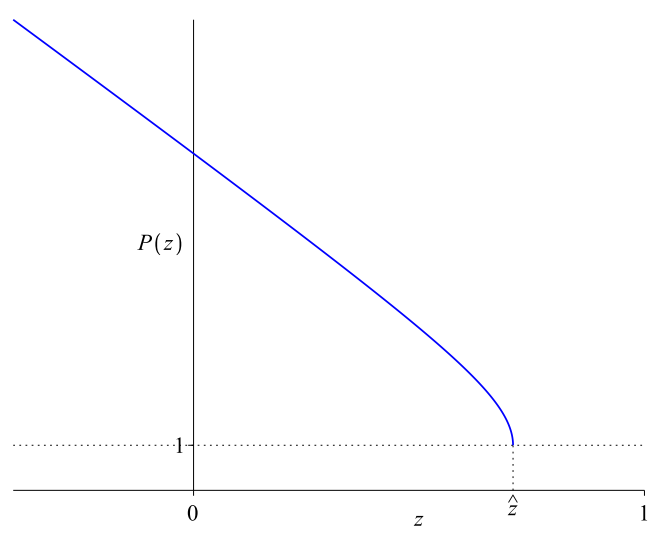

Fig. 4 The Poincaré map $P$ for $\gamma<0$ and $\beta>0$. Notice that $P(z)$ is only defined for $z \leq \hat{z}<1$ and $P(z) \geq 1$. Here $\hat{z}>0$, but negative values for $\hat{z}$ are allowed.

map $P$ is explicitly obtained,

$P(z)=1-e^{\gamma \pi}(z-1), \quad z<1$.

In order to look for crossing limit cycles, we solve the equation $z=-P(z)$ whose solution is

$z=\frac{1+e^{\gamma \pi}}{1-e^{\gamma \pi}}=\operatorname{coth}\left(\frac{\gamma \pi}{2}\right)<-1$.

Moreover, the modulus of the derivative of Poincaré map verifies $\left|P^{\prime}(z)\right|=e^{\gamma \pi}<1$. Hence, for $\beta=0$ and $Q>1 / 2$, one gets that the system has only one crossing limit cycle, which is stable.

\subsubsection{The case $\beta>0$}

Assuming $\beta>0$ and solving (13), the following parametric representation for the Poincaré map $z_{1}(\theta)=P(z(\theta))$ is obtained.

$z(\theta)=1+\bar{x}_{2}\left(\gamma+\frac{e^{-\gamma \theta}-\cos \theta}{\sin \theta}\right)=1-\frac{2 \beta \gamma e^{-\gamma \theta} \varphi_{\gamma}(\theta)}{\left(1+\gamma^{2}\right) \sin \theta}$, 
$P(z(\theta))=1+\bar{x}_{2}\left(\gamma-\frac{e^{\gamma \theta}-\cos \theta}{\sin \theta}\right)=1+\frac{2 \beta \gamma \gamma^{\gamma \theta} \varphi_{-\gamma}(\theta)}{\left(1+\gamma^{2}\right) \sin \theta}$,

where $\pi<\theta \leq \hat{\theta}$, being $\varphi_{-\gamma}(\hat{\theta})=0$. Obviously, variables $z$ and $P(z)$ also depend on parameters $\beta$ and $\gamma$; such a dependence is made explicit only when it is oportune. The map $P$ is defined for $z \leq \hat{z}=z(\hat{\theta})<1$, with $P(z) \geq 1$, and $P(z) \rightarrow \infty$ for $z \rightarrow-\infty$. An illustrative plot of the map $P(z)$ is given in Fig. 4.

Direct computations show that for $\pi<\theta \leq \hat{\theta}$, the two first derivatives of $P$ are

$P^{\prime}(z)=-\frac{\varphi_{\gamma}(\theta)}{\varphi_{\gamma}(-\theta)}=\frac{(z-1) e^{2 \gamma \theta}}{P(z)-1}<0$,

$P^{\prime \prime}(z)=\frac{8 \beta^{2} \gamma^{2}(\sinh (\gamma \theta)-\gamma \sin \theta) e^{3 \gamma \theta}}{\left(1+\gamma^{2}\right)(P(z)-1)^{3}}<0$.

Moreover, when $z \rightarrow-\infty$, then $\theta \rightarrow \pi$ and when $z \rightarrow \hat{z}$, then $\theta \rightarrow \hat{\theta}$. Therefore, we have

$\lim _{z \rightarrow-\infty} P(z)=\infty$,

$\lim _{z \rightarrow-\infty} P^{\prime}(z)=-e^{\gamma \pi}, \quad \lim _{z \rightarrow \hat{z}} P^{\prime}(z)=-\infty$.

Now, we can state the following fact. For $\beta>0$, system (11) can have at most two crossing limit cycles because the map $P$ is concave down and so, its graph meets the secondary diagonal at most in two points. The existence and stability of these crossing limit cycles are studied in Subsection 3.4 after analyzing the closed curves containing the full sliding set or a part of it.

\subsection{The sliding invariant closed curves}

Invariant closed curves containing points in the sliding set $\Sigma^{s}$ will be called sliding closed curves. Since orbits do not arrive at the repulsive sliding segment in forward time, the only possible sliding invariant curves occur in backward time and they must contain at least one of the tangency points.

When $\beta=0$, from (12) every point in the sliding set is a pseudo-equilibrium point and consequently sliding invariant closed curves cannot exist.

To determine sliding limit cycles when $\beta>0$, the orbit through the tangency point $\mathbf{x}_{B}^{+}=(1,0)$ is considered. Next, assuming $\gamma<0$ and taking $\beta$ as the bifurcation parameter, our interest is to compute the value of $z$, such that $P(z)=$ 1. From expression (18) the condition $\varphi_{-\gamma}(\theta)=0$ must be satisfied, and so $\theta=\hat{\theta}$, see (15). After some algebra, we get

$\hat{z}=P^{-1}(1)=z(\hat{\theta})=1-\frac{2 \beta \gamma}{\gamma+\cot \hat{\theta}}=1-\frac{\beta}{\beta_{h c}(\gamma)}$, where $\beta_{h c}(\gamma)$ is a function that can be defined as follows

$$
\beta_{h c}(\gamma)=\frac{1}{2}+\frac{\cot \hat{\theta}}{2 \gamma}=\frac{e^{\gamma \hat{\theta}}}{2 \gamma \sin \hat{\theta}}=\frac{1}{2\left(1-e^{-\gamma \hat{\theta}} \cos \hat{\theta}\right)} .
$$

The equivalence among expressions in (24) comes from (15).

Different scenarios can appear as it is shown in the next proposition.

Proposition 3 Assuming $\gamma<0$ in system (8)-(9), and taking $\beta_{h c}(\gamma)$ as in (24), the following statements hold.

(a) The function $\beta_{h c}(\gamma)$ is positive and increasing with $\gamma$.

(b) If $\beta=0$, then sliding limit cycles do no exist.

(c) If $0<\beta<\beta_{h c}(\gamma)$, then there exists a symmetric pair of unstable sliding limit cycles, each one of them living in only one zone.

(d) If $\beta=\beta_{h c}(\gamma)$, then the above limit cycles become into a symmetric pair of unstable sliding homoclinic connections to the origin.

(e) If $\beta_{h c}(\gamma)<\beta<2 \beta_{h c}(\gamma)$, then there exists one unstable sliding limit cycle being symmetrical with respect to the origin.

Proof The conditions $\pi<\hat{\theta}<2 \pi$ and $\gamma<0$ imply $\gamma \sin \hat{\theta}>$ 0 , and then $\beta_{h c}>0$. Also, by computing the derivative

$\frac{\mathrm{d} \beta_{h c}}{\mathrm{~d} \gamma}=\frac{\partial \beta_{h c}}{\partial \hat{\theta}} \frac{\mathrm{d} \hat{\theta}}{\mathrm{d} \gamma}+\frac{\partial \beta_{h c}}{\partial \gamma}$,

we get

$\frac{\mathrm{d} \beta_{h c}}{\mathrm{~d} \gamma}=\frac{\gamma(\hat{\theta}-\sin \hat{\theta} \cos \hat{\theta})-\sin ^{2} \hat{\theta}}{2 e^{\gamma \hat{\theta}} \sin \hat{\theta}\left(\gamma^{2}+1\right)\left(1-e^{-\gamma \hat{\theta}} \cos \hat{\theta}\right)^{2}}$,

and taking into account that $\hat{\theta}-\sin \hat{\theta} \cos \hat{\theta}>0$, it is concluded that $\beta_{h c}^{\prime}(\gamma)>0$, and so $\beta_{h c}(\gamma)$ is increasing.

The orbit in backward time through the tangency point $\mathbf{x}_{B}^{+}$evolves in the region $\Sigma^{+}$and after surrounding the equilibrium point $\overline{\mathbf{x}}^{+}$, arrives at the switching manifold at the point $(\hat{z}, 0)$ and the following cases arise.

If $\beta=0$, then the sliding set is full of pseudo-equilibrium what precludes the existence of any sliding motion.

If $0<\beta<\beta_{h c}(\gamma)$, then from (23), it is found that $0<\hat{z}<$ 1. In this case, the orbit continues in backward time from the point $(\hat{z}, 0)$ sliding towards the point $\mathbf{x}_{B}^{+}$making one sliding invariant closed curve. The symmetry imposes the existence of another invariant closed curve on the other side of $\Sigma$.

When $\beta=\beta_{h c}(\gamma)$, then $\hat{z}=0$, i.e., the orbit arrives at the pseudo-saddle located at the origin. Since the sliding dynamics is repulsive towards the point $\mathbf{x}_{B}^{+}$, there is a homoclinic connection to the origin. The symmetry imposes the existence of another homoclinic connection to the origin on the other side of $\Sigma$.

When $\beta_{h c}(\gamma)<\beta<2 \beta_{h c}(\gamma)$, then $-1<\hat{z}<0$. From the point $(\hat{z}, 0)$, the orbit continues sliding in backward time towards the tangency point $\mathbf{x}_{B}^{-}=(-1,0)$. Then the symmetry 
imposes the closing of the orbit at the point $\mathbf{x}_{B}^{+}$, making a sliding limit cycle living in the two zones.

In any case, the invariant closed curve is unstable because it contains a repulsive sliding segment.

\subsection{The crossing limit cycles}

In this section, the existence of crossing limit cycles is analyzed by fixing the parameter $\gamma<0$ and taking $\beta$ as the bifurcation parameter.

As shown before, crossing limit cycles are determined by points $z \leq-1$ satisfying $P(z)=-z$. Thus, they correspond to the zeros of the function

$g(z)=z+P(z)$.

The dependence for the function $g$ of the parameters will be sometimes emphasized by introducing the notation

$g(z(\theta ; \beta, \gamma))=z(\theta ; \beta, \gamma)+P(z(\theta ; \beta, \gamma))$,

which from (17)-(18) can be written as,

$g(z(\theta ; \beta, \gamma))=2-\frac{4 \beta \gamma}{1+\gamma^{2}}\left(\gamma-\frac{\sinh (\gamma \theta)}{\sin \theta}\right)$.

Next, some properties of the function $g$ are established, see Fig. 5.

(a) The function $g$ is defined for $z \leq \hat{z}=P^{-1}(1)$. Furthermore, if $z \rightarrow-\infty$, then $\theta \rightarrow \pi$ and from (26),

$\lim _{z \rightarrow-\infty} g(z)=-\infty$.

In addition, taking into account (23), it is found

$g(\hat{z})=\hat{z}+P(\hat{z})=\hat{z}+1=2-\frac{\beta}{\beta_{h c}(\gamma)}$.

(b) From (19)-(22) we deduce $g^{\prime \prime}(z)<0$ and so, the derivative $g^{\prime}(z)$ monotonically decreases when $-\infty<z<\hat{z}$, and furthermore,

$\lim _{z \rightarrow-\infty} g^{\prime}(z)=1-e^{\gamma \pi}>g^{\prime}(z)>\lim _{z \rightarrow \hat{z}} g^{\prime}(z)=-\infty$.

Since $\gamma<0$, then $1-e^{\gamma \pi}>0$, so that there exists one point $z_{M}(\beta)$ with $g^{\prime}\left(z_{M}(\beta)\right)=1+P^{\prime}\left(z_{M}(\beta)\right)=0$. Thus, the function $g$ is concave down and its maximum value is $g\left(z_{M}(\beta)\right)$.

(c) Since the derivative of the Poincare map $P$ satisfies the condition $P^{\prime}\left(z_{M}(\beta)\right)=-1$, the flight time $\theta_{M} \in(\pi, \hat{\theta})$ corresponding to the point $z_{M}(\beta)$ is determined by the relation $\varphi_{\gamma}\left(-\theta_{M}\right)=\varphi_{\gamma}\left(\theta_{M}\right)$, see (19) and Fig. 3, or equivalently by the equation

$\gamma \operatorname{coth}\left(\gamma \theta_{M}\right)-\cot \theta_{M}=0$.

Note that $\gamma \operatorname{coth}\left(\gamma \theta_{M}\right)>0$, so that $\theta_{M} \in(\pi, 3 \pi / 2)$. Then, the maximum value of function $g$ is obtained by taking $\theta=\theta_{M}$ in (26). Note that $\theta_{M}$ depends only on $\gamma$, so that $z_{M}(\beta, \gamma)=z\left(\theta_{M} ; \beta, \gamma\right)$, see (17). (d) It should be pointed out that

$$
\frac{\partial g(z(\theta ; \beta, \gamma))}{\partial \beta}=\frac{-4 \gamma}{1+\gamma^{2}}\left(\gamma-\frac{\sinh (\gamma \theta)}{\sin \theta}\right)<0 .
$$

The function $\theta_{M}=\theta_{M}(\gamma)$ which is implicitly defined from equation (28) has the first derivative

$\frac{\mathrm{d} \theta_{M}}{\mathrm{~d} \gamma}=\frac{2 \gamma \theta-\sinh (2 \gamma \theta)}{2 \sinh ^{2}(\gamma \theta)\left(1+\gamma^{2}\right)}>0$,

and then the function $\theta_{M}(\gamma)$ is increasing. Also, from (28) it can be shown that when $\gamma \rightarrow-\infty$, then $\theta_{M} \rightarrow \pi$ and when $\gamma \rightarrow 0$, then $\theta_{M}$ tends to $4.493409 \ldots$, which is the only root of the equation $\theta \cot \theta=1$ in the interval $(\pi, 3 \pi / 2)$.

Since any crossing limit cycle is symmetric with respect to the origin, the minimum absolute value of its intersection with the switching manifold $\Sigma$ is clearly 1 , thus corresponding to a critical cycle that links the two endpoints $\mathbf{x}_{B}^{+}$and $\mathbf{x}_{B}^{-}$of the sliding set. Let this cycle be called critical crossing limit cycle (CC limit cycle, for short). For the sake of convenience, let us introduce the function

$\beta_{c c}(\gamma)=2 \beta_{h c}(\gamma)$

Now, the following results about crossing limit cycles are given, see Fig. 5. Note that the new introduced value $\beta_{s n}(\gamma)$ corresponds to the case when the maximum value of function $g$ vanishes.

Proposition 4 Assuming $\gamma<0$ in system (8)-(9), there exists a positive function

$\beta_{s n}(\gamma)=\frac{\left(1+\gamma^{2}\right) \sin \theta_{M}}{2 \gamma\left(\gamma \sin \theta_{M}-\sinh \left(\gamma \theta_{M}\right)\right)}$,

where $\theta_{M}$ is the only root of equation (28) in the interval $(\pi, 3 \pi / 2)$ such that

$g\left(z_{M}\left(\beta_{s n}\right)\right)=0$

is satisfied and the following statements hold.

(a) The function $\beta_{s n}(\gamma)$ is positive and increasing with $\gamma$.

(b) If $\beta>\beta_{s n}(\gamma)$ then there are no crossing limit cycles.

(c) If $\beta=\beta_{s n}(\gamma)$ there exists one semi-stable crossing limit cycle.

(d) If $\beta_{c c}(\gamma)<\beta<\beta_{s n}(\gamma)$ then there exist two crossing limit cycles. The inner is unstable being the outer stable.

(e) If $\beta=\beta_{c c}(\gamma)$ then there exist one unstable CC limit cycle and one stable crossing limit cycle.

(f) If $\beta<\beta_{c c}(\gamma)$ then there exists one stable crossing limit cycle.

Proof Taking $\theta=\theta_{M}$ and $\beta=\beta_{s n}$ in expression (26), the equality $g\left(z_{M}\left(\beta_{s n}\right)\right)=0$ is obtained. 


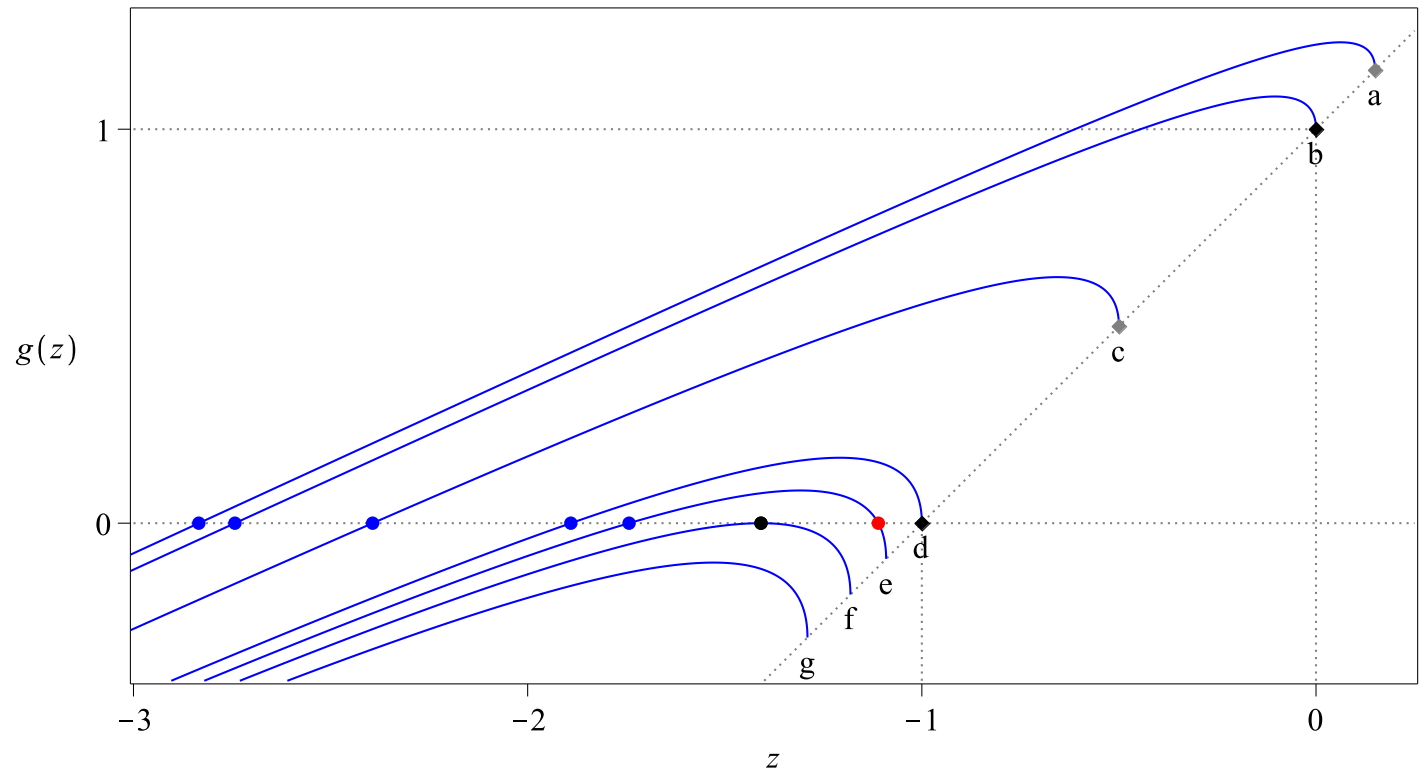

Fig. 5 Different graphs of the function $g(z)$ for a fixed value of parameter $\gamma$ and different values of the parameter $\beta$ such that the dynamics corresponds to the cases (a)-(g) summarized in Theorem 1 . Note that each graph is defined for $z \leq \hat{z}(\beta, \gamma)$ with $g(\hat{z})=1+\hat{z}$. Circle points stand for crossing limit cycles, the blue, red and black ones correspond to stable, unstable and semi-stable crossing limit cycles respectively. Diamond endpoints whose coordinates are $(\hat{z}, 1+\hat{z})$ with $\hat{z}>-1$ represent sliding invariant curves. In particular, the critical crossing cycle corresponds to $\hat{z}=-1$ and the homoclinic connection corresponds to $\hat{z}=0$ (see black diamond endpoints).

Since the conditions $\gamma<0$ and $\pi<\theta_{M}<3 \pi / 2$ hold, the inequality $\beta_{s n}>0$ follows. Also, by computing the derivative

$\frac{\mathrm{d} \beta_{s n}}{\mathrm{~d} \gamma}=\frac{\partial \beta_{s n}}{\partial \theta_{M}} \frac{\mathrm{d} \theta_{M}}{\mathrm{~d} \gamma}+\frac{\partial \beta_{s n}}{\partial \gamma}$

and having in mind equation (28), we get $\frac{\partial \beta_{s n}}{\partial \theta_{M}}=0$, so

$\frac{\mathrm{d} \beta_{s n}}{\mathrm{~d} \gamma}=\sin \theta_{M} \frac{\Psi_{1}\left(\gamma, \theta_{M}\right)+\gamma^{2} \Psi_{2}\left(\gamma, \theta_{M}\right)}{2 \gamma^{2}\left(\gamma \sin \theta_{M}-\sinh \left(\gamma \theta_{M}\right)\right)^{2}}$,

where

$\Psi_{1}\left(\gamma, \theta_{M}\right)=\sinh \left(\gamma \theta_{M}\right)+\gamma \theta_{M} \cosh \left(\gamma \theta_{M}\right)-2 \gamma \sin \theta_{M}<0$

and

$\Psi_{2}\left(\gamma, \theta_{M}\right)=\gamma \theta_{M} \cosh \left(\gamma \theta_{M}\right)-\sinh \left(\gamma \theta_{M}\right)<0$

Then, $\beta_{s n}^{\prime}(\gamma)>0$, and statement (a) follows.

To show the remaining statements, we need to compute the number of zeros of function $g$ defined in (25). This computation will be done by studying the sign of the maximum value of the function $g$, that is $g\left(z_{M}\right)$ and the value of the function $g$ at the endpoint of the definition interval $(-\infty, \hat{z}]$ of function $g$, that is $g(\hat{z})$, see Fig. 5

Taking $\theta=\theta_{M}$ in (29), it turns out that

$\frac{\mathrm{d} g\left(z_{M}(\beta)\right)}{\mathrm{d} \beta}<0$, hence, $g\left(z_{M}(\beta)\right)$ is decreasing with $\beta$. Then, $g\left(z_{M}(\beta)\right)>0$ for $\beta<\beta_{s n}$ and $g\left(z_{M}(\beta)\right)<0$ for $\beta>\beta_{s n}$.

If $\beta>\beta_{s n}(\gamma)$, then $g(z) \leq g\left(z_{M}(\beta)\right)<0$, the function $g$ does not vanish and there are no crossing limit cycles.

If $\beta=\beta_{s n}(\gamma)$, then $g(z)<g\left(z_{M}\left(\beta_{s n}\right)\right)=0$ for $z \neq z_{M}\left(\beta_{s n}\right)$ and the equality $P^{\prime}\left(z_{M}\left(\beta_{s n}\right)\right)=-1$ hold. Hence the point $z_{M}\left(\beta_{s n}\right)$ corresponds to the only crossing limit cycle which is semi-stable.

If $\beta<\beta_{s n}(\gamma)$ then $g\left(z_{M}\left(\beta_{s n}\right)\right)>0$. Having in mind that $g(z) \rightarrow-\infty$ when $z \rightarrow-\infty$ and $g^{\prime}(z)>0$ for $z<z_{M}\left(\beta_{s n}\right)$, we conclude the existence of a unique point $z_{s}(\beta)<z_{M}(\beta)$ such that $g\left(z_{s}(\beta)\right)=0$. Moreover, the derivative of the Poincare map verifies $0>P^{\prime}\left(z_{s}\right)>P^{\prime}\left(z_{M}\right)=-1$, then the existence of a stable crossing limit cycle is proved.

Next, the existence of another crossing limit cycle depending on the sign of $g(\hat{z})$ is determined. From (27) it follows that $g(\hat{z}(\beta))=0$ for $\beta=\beta_{c c}$ along with $g(\hat{z}(\beta))>0$ for $\beta<\beta_{c c}$ and $g(\hat{z}(\beta))<0$ for $\beta>\beta_{c c}$.

If $\beta_{c c}(\gamma)<\beta<\beta_{s n}(\gamma)$ then $g\left(z_{M}(\beta)\right)>0, g(\hat{z}(\beta))<0$ and $g^{\prime}(z)<0$ for $z_{M}(\beta)<\hat{z}(\beta)$. Then there exists a unique point $z_{u}(\beta)>z_{M}(\beta)$ such that $g\left(z_{u}(\beta)\right)=0$ and the derivative of the Poincaré map verifies $P^{\prime}\left(z_{M}\right)=-1<P^{\prime}\left(z_{u}\right)$. Then there is a crossing limit cycle which is unstable, in addition to the above shown stable crossing limit cycle, because $\beta<\beta_{\text {sn }}(\gamma)$.

If $\beta=\beta_{c c}(\gamma)$ then $g(\hat{z})=0$ with $\hat{z}=-1$. Hence, in addition to the stable limit cycle existing for $\beta<\beta_{s n}(\gamma)$, a limit cycle linking the points $\mathbf{x}_{B}^{ \pm}$appears. 


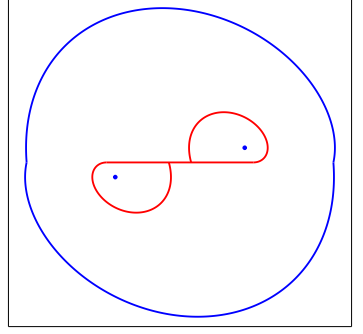

(a) $\beta<\beta_{h c}(\gamma)$

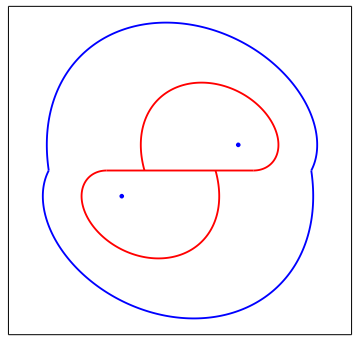

(c) $\beta_{h c}(\gamma)<\beta<\beta_{c c}(\gamma)$

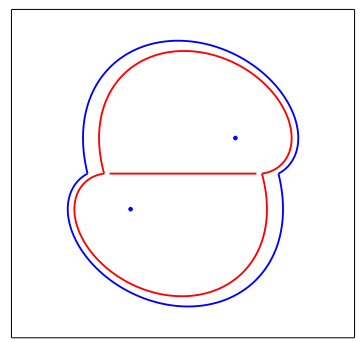

(e) $\beta_{c c}(\gamma)<\beta<\beta_{s n}(\gamma)$

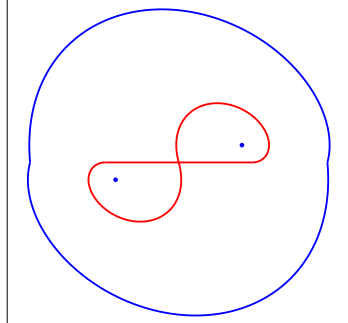

(b) $\beta=\beta_{h c}(\gamma)$

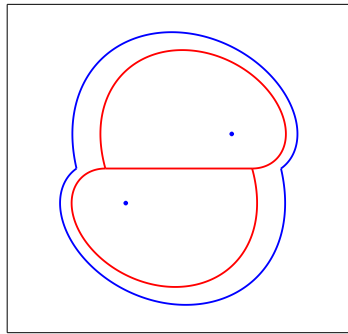

(d) $\beta=\beta_{c c}(\gamma)$

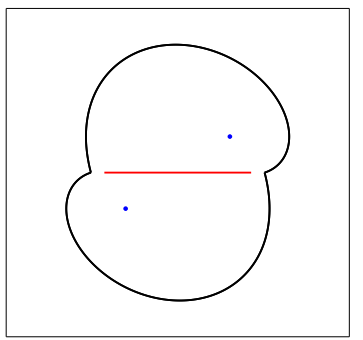

(f) $\beta=\beta_{s n}(\gamma)$
Fig. 6 Limit sets for parameter $\gamma$ fixed and parameter $\beta$ specified in the caption. The equilibrium points and the outer stable limit cycle are depicted in blue color and the unstable cycles in red color. The black cycle in (f) corresponds to the non hyperbolic limit cycle at the saddlenode bifurcation. The red straight line corresponds to the sliding set $\Sigma^{s}$.

If $\beta<\beta_{c c}(\gamma)$ then $g(\hat{z}(\beta))>0$, hence only the stable crossing limit cycle exists.

Remark 1 The stable crossing limit cycle that exists for $\beta<$ $\beta_{s n}$, corresponds to a value $z_{s}\left(\theta_{s} ; \beta\right)<z_{M}(\beta)$, and its period $2 \theta_{s}$ satisfies $2 \pi<2 \theta_{s}<2 \theta_{M}$.

The unstable crossing limit cycle exists for $\beta_{c c}<\beta<$ $\beta_{s n}$, it corresponds to a value $z_{u}\left(\theta_{u} ; \beta\right)>z_{M}(\beta)$, and its period $2 \theta_{u}$ satisfies $2 \theta_{M}<2 \theta_{u}<2 \hat{\theta}$.

See Fig. 8 below for a diagram of relevant flight times $\hat{\theta}$, $\theta_{M}, \theta_{s}$ and $\theta_{u}$, regarding several parameter configurations.
3.5 Limit cycle bifurcations in resonant LC inverters

From the above analysis and regarding the existence of closed invariant curves, the following result is a direct consequence of propositions 3 and 4 .

Theorem 1 Assuming $\gamma<0$ in system (8)-(9), the following statements hold.

(a) If $0<\beta<\beta_{h c}(\gamma)$ then there exist one stable crossing limit cycle and two unstable sliding limit cycles.

(b) If $\beta=\beta_{h c}(\gamma)$ then there exist one stable crossing limit cycle and two homoclinic connections to the origin.

(c) If $\beta_{h c}(\gamma)<\beta<\beta_{c c}(\gamma)$ then there exist one stable crossing limit cycle and one unstable sliding limit cycle.

(d) If $\beta=\beta_{c c}(\gamma)$ then there exist one stable crossing limit cycle and one unstable critical crossing limit cycle.

(e) If $\beta_{s n}(\gamma)<\beta<\beta_{c c}(\gamma)$ then there exist two crossing limit cycles having opposite stability.

(f) If $\beta=\beta_{s n}(\gamma)$ then there is one crossing limit cycle which is semi-stable.

(g) If $\beta>\beta_{s n}(\gamma)$ then there are no crossing limit cycles.

The function $g(z)$ is represented in Fig. 5 for a fixed value of the parameter $\gamma$ and different values of the parameter $\beta$, illustrating the seven cases summarized in Theorem 1. Furthermore, the limit sets in the phase plane are depicted in Fig. 6 for the first six cases described in Theorem 1. Observe the crucial role that the abscissa of the endpoint $\hat{z}$ plays in determining limit cycles. In fact, the following cases arise:

(a) If $0<\hat{z}<1$, then there exist one stable crossing limit cycle and two sliding limit cycles, see curve (a) in Fig. 5 and Fig. 6 (a).

(b) If $\hat{z}=0$, then there exist one stable crossing limit cycle and a double homoclinic connection, see curve (b) in Fig. 5 and Fig. 6 (b).

(c) If $-1<\hat{z}<0$, then there exist one stable crossing limit cycle and one sliding limit cycle, see curve (c) in Fig. 5 and Fig. 6 (c).

(d) If $\hat{z}=-1$, then there exist one stable crossing limit cycle and one unstable CC limit cycle, see curve (d) in Fig. 5 and Fig. 6 (d).

(e) If $\hat{z}<-1$, then three different cases arise depending on the value of $\beta$. These are two crossing limit cycles with opposite stabilities as for curve (e) in Fig. 5 or Fig. 6 (e), one semi-stable crossing limit cycle as for curve (f) in Fig. 5 or Fig. 6 (f), and no limit cycles in the case of curve $(\mathrm{g})$ in Fig. 5.

Notice that the unstable closed curves determine the attraction basin of the stable equilibria.

Let us remark that for cases (a), (c), (e) and (g) the dynamics is structurally stable, that is, it remains qualitatively unchanged under small parameter variations. Conversely, the transition cases (b), (d) and (f) stand for the double homoclinic saddle connection, the critical crossing cycle and the 


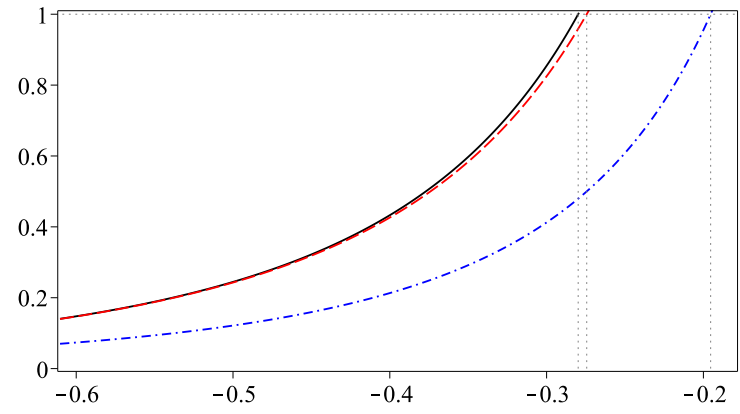

(a) $\beta_{s n}, \beta_{c c}$ and $\beta_{h c}$ versus $\gamma$

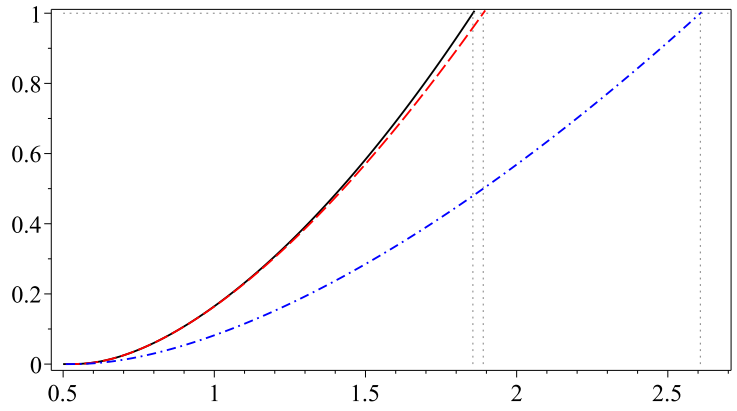

(b) $\beta_{s n}, \beta_{c c}$ and $\beta_{h c}$ versus $Q$

Fig. 7 Left panel: Graphs of the functions $\beta_{s n}(\gamma), \beta_{c c}(\gamma)$ and $\beta_{h c}(\gamma)$. Right panel: Graph of the functions $\beta_{s n}(Q), \beta_{c c}(Q)$ and $\beta_{h c}(Q)$. In both panels, the solid black line stands for $\beta_{s n}$, the dashed red line does for $\beta_{c c}$ and the dashed dotted blue line does for $\beta_{h c}$; also the horizontal line $\beta=1$ marks the maximum value that this parameter can reach and the vertical lines give account of the corresponding minimum values of parameter $\gamma$ (left panel) or the minimum values of quality factor $Q$ (right panel) in order to avoid the homoclinic connection, critical crossing and saddle-node bifurcation for any valid value of parameter $\beta$.

saddle-node bifurcation for cycles, respectively. In the left panel of Fig. 7, the three curves plotted in the parameter plane stand for these three codimension one bifurcations, that is $\beta_{h c}(\gamma), \beta_{c c}(\gamma)$ and $\beta_{s n}(\gamma)$. These critical values of $\beta$ are also represented in the right panel of Fig. 7, this time versus the parameter $Q$.

In practice, it is more convenient to take $\gamma$ as the main variable parameter. Having in mind the monotonicity of the functions $\beta_{h c}(\gamma), \beta_{c c}(\gamma)$ and $\beta_{s n}(\gamma)$, their respective inverse functions $\gamma_{h c}(\beta), \gamma_{c c}(\beta)$ and $\gamma_{s n}(\beta)$ can be defined. Under this point of view, the crossing limit cycle bifurcations are described as follows.

Remark 2 Assuming $\beta$ fixed and $\gamma$ as the variable parameter, the limit cycle bifurcations are as follows.

(a) A homoclinic bifurcation at $\gamma=\gamma_{h c}(\beta)$, such that the two sliding limit cycles existing for $\gamma_{h c}(\beta)<\gamma$ become one sliding limit cycle, which surrounds the origin, for $\gamma_{c c}(\beta)<\gamma<\gamma_{h c}(\beta)$.

(b) A critical crossing bifurcation at $\gamma=\gamma_{c c}(\beta)$ such that the sliding limit cycle existing for $\gamma_{c c}(\beta)<\gamma<\gamma_{h c}(\beta)$ becomes a critical crossing limit cycle and afterwards a crossing limit cycle for $\gamma_{s n}(\beta)<\gamma<\gamma_{c c}(\beta)$.

(c) A saddle-node bifurcation at $\gamma=\gamma_{s n}(\beta)$, such that the two crossing limit cycles with opposite stabilities existing for $\gamma_{s n}(\beta)<\gamma<\gamma_{c c}(\beta)$ becomes one semi-stable crossing limit cycle and afterwards disappear for $\gamma<$ $\gamma_{s n}(\beta)$.

Consequently, to guaranty the existence of a stable oscillation, the mandatory condition in a practical application is $\gamma>\gamma_{s n}(\beta)$. However, it must be pointed out that since the system has also two stable equilibrium points, it is desirable that their attraction basins do not include the origin in order to facilitate the starting up process. Hence, to meet this stronger requirement, the condition $\gamma>\gamma_{h c}(\beta)$ should be fulfilled and so, under such a restriction there is only one crossing limit cycle which is stable.

Let us also mention that due to the physical restriction $0<\beta \leq 1$, the homoclinic connection, the critical crossing cycle and the saddle-node bifurcations cannot be given in the inverter for values of the parameter $\gamma$ greater than $\gamma_{h c}(1)=-0.195379 \ldots, \gamma_{c c}(1)=-0.274411 \ldots$ and $\gamma_{s n}(1)=$ $-0.279860 \ldots$, respectively. These critical values can been computed as follows.

\section{Proposition 5 The following statements hold.}

(a) The value $\gamma_{h c}(1)$ is given by $\gamma_{h c}(1)=\cot \hat{\theta}$, where $\hat{\theta}$ is the only root of the equation

$e^{\theta \cot \theta}=2 \cos \theta$

in the interval $(\pi, 2 \pi)$.

(b) The value $\gamma_{c c}(1)$ is the only root of the equation

$e^{\gamma \frac{3 \pi}{2}}+\gamma=0$

(c) The value $\gamma_{s n}(1)$ is the only value for the parameter $\gamma$ which is solution of the system of equations

$$
\left(\gamma^{2}-1\right) \sin \theta=2 \gamma \sinh \theta,
$$$$
\cot \theta=\gamma \operatorname{coth}(\gamma \theta)
$$

for $\theta \in(\pi, 3 \pi / 2)$.

Proof The equality $\beta_{h c}=1$ implies $\gamma=\cot \hat{\theta}$, see (24). Then, by putting $\gamma=\cot \hat{\theta}$ in (15), statement (a) follows.

From the condition $\beta_{c c}=2 \beta_{h c}=1$, it follows $\cot \hat{\theta}=0$ with $\pi<\hat{\theta}<2 \pi$, that is $\hat{\theta}=3 \pi / 2$, see (24). Then, statement (b) is straightforward by replacing $\hat{\theta}=3 \pi / 2$ in (15).

Imposing $\beta_{s n}=1$ in expression (30) and using expression (28) which determines $\theta_{M}$, statement (c) follows. 


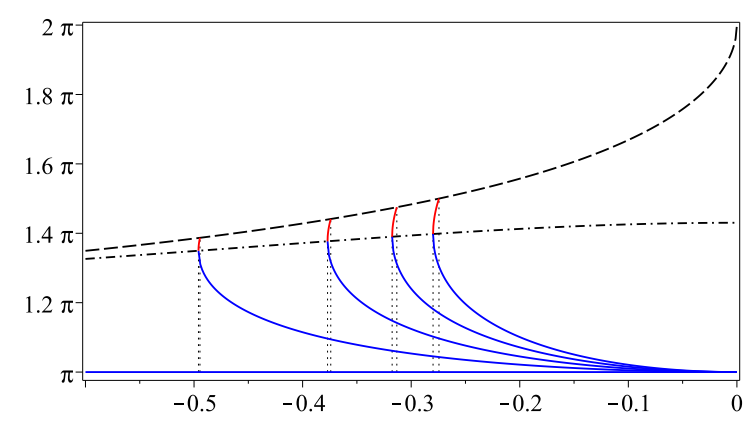

Fig. 8 Plots of the functions $\theta_{s}(\gamma)$ in solid blue, $\theta_{u}(\gamma)$ in solid red, $\hat{\theta}(\gamma)$ in dash black and $\theta_{M}(\gamma)$ in dash-dotted black versus parameter $\gamma$, with parameter $\beta \in \mathscr{B}$. Note that $\theta_{s}=\pi$ when $\beta=0$ and in the other cases, the four sets of two vertical lines indicate the smooth saddle-node and the critical crossing bifurcations for cycles at $\gamma_{s n}$ and $\gamma_{c c}$ respectively.

\section{Oscillation features regarding applications}

This section is addressed to compute the frequency and size of the stable crossing limit cycle by considering $\gamma$ as the main variable parameter. Notice that in the context of applications, the system is expected to behave properly under some specified load range. In practice a variation in the load resistor, $R_{o s}$ or $R_{o p}$, would produce a significant change in the parameter $\gamma$, but minor changes in the parameter $\beta$.

According to Remarks 1-2, the stable limit cycle exists for $\gamma_{s n}(\beta)<\gamma<0$ with a half-period $\theta_{s}$ satisfying $\pi<\theta_{s}<$ $\theta_{M}$.

Once computed $\theta_{s}$ as the smaller value for $\theta$ vanishing (26), from the time changes used in propositions 1 and 2 we can compute the period $T$ (or the corresponding frequency $\omega=2 \pi / T$ ) of the stable oscillation in system (1). Since the normalized time $\theta$ in system (8)-(9) is related to the real time $t$ in system (1)-(2) by the relation $\theta=\omega_{0} v t$, we obtain $T=2 \theta_{s} /\left(v \omega_{0}\right)$. Then, the relative frequency $\omega_{r}$ with respect to the natural frequency $\omega_{0}$ is

$\omega_{r}=\frac{\omega}{\omega_{0}}=\frac{\pi v}{\theta_{s}}=\frac{\pi}{\theta_{s} \sqrt{1+\gamma^{2}}}=\frac{\pi}{\theta_{s}} \sqrt{1-\frac{1}{4 Q^{2}}}$.

In order to evaluate the size of the oscillation, let us use the absolute value of the intersection of the stable crossing limit cycle with the switching manifold $\Sigma$, which will be denoted as $\Sigma$-amplitude, for short $a_{\Sigma}$. Then $a_{\Sigma}=P\left(z\left(\theta_{s}\right)\right)=$ $\left|z\left(\theta_{s}\right)\right|>0$, and from (17)-(18),

$a_{\Sigma}=\frac{P\left(z\left(\theta_{s}\right)\right)+\left|z\left(\theta_{s}\right)\right|}{2}=2 \beta \gamma \frac{\cosh \left(\gamma \theta_{s}\right)-\cos \theta_{s}}{\left(1+\gamma^{2}\right) \sin \theta_{s}}$.

To illustrate the parameter dependence of these magnitudes, they will be represented as function of either $\gamma$ or $Q$, and $\beta$ will be chosen within the set

$\mathscr{B}=\{0,1 / 4,1 / 2,3 / 4,1\}$.

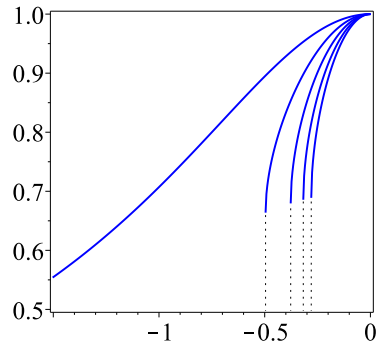

(a) $\omega_{r}(\gamma)$

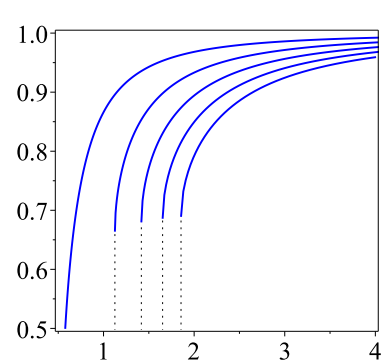

(b) $\omega_{r}(Q)$
Fig. 9 Diagrams representing the relative frequency $\omega_{r}$ versus parameter $\gamma$ in (a) and versus $Q$ in (b) with parameter $\beta \in \mathscr{B}$. For $\beta>0$, the vertical lines indicate the saddle-node bifurcation.

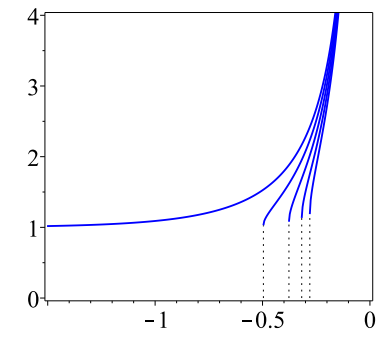

(a) $a_{\Sigma}(\gamma)$

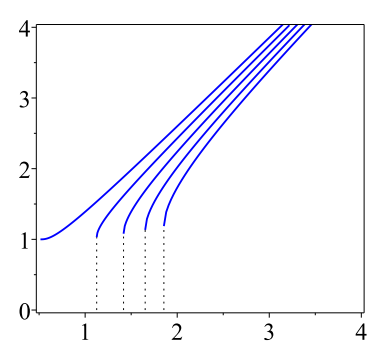

(b) $a_{\Sigma}(Q)$
Fig. 10 Diagrams representing the $\Sigma$-amplitude $\left(a_{\Sigma}\right)$ of the stable crossing limit cycle versus parameter $\gamma$ in (a) and versus $Q$ in (b) with parameter $\beta \in \mathscr{B}$. For $\beta>0$, vertical lines indicate the saddle-node bifurcation.

In Fig. 8, the half-period $\theta_{s}$ is shown as a function of the parameter $\gamma$, for $\beta \in \mathscr{B}$. To make clearer this representation, times $\theta_{u}, \hat{\theta}$ and $\theta_{M}$ are also included. In each case, excepting $\beta=0$ (and hence $\theta_{s}=\pi$ ), the two vertical dotted lines indicate the saddle-node, $\gamma_{s n}(\beta)$, and the critical crossing, $\gamma_{c c}(\beta)$, bifurcations for limit cycles. Let us recall that both functions $\gamma_{s n}(\beta)$ and $\gamma_{c c}(\beta)$ increase with $\beta$ and that these bifurcations (and also the homoclinic connection) are not possible for $\beta=0$.

In the last diagram sets given in Figs. 9-10, the relative frequency $\omega_{r}$ and the $\Sigma$-amplitude $\left(a_{\Sigma}\right)$ are shown versus the parameter $\gamma$ in the left panels and versus the parameter $Q$ in the right panels, for $\beta \in \mathscr{B}$. The four vertical dotted lines, which are located at $\gamma_{s n}(\beta)$ or at the corresponding value for $Q$ in the right panels, indicate the saddle-node bifurcation for crossing cycles.

Note that when $\gamma$ approaches 0 and accordingly $Q$ increases, the relative frequency $\omega_{r}$ tends to 1 and $a_{\Sigma}$ growths as much as desired. 


\section{Conclusions}

Emerging applications of power electronics have recently motivated the use of soft switching self oscillating resonant parallel and series converters because their advantages with respect to their hard switching counterparts. The design and the stability analysis of these systems are challenging tasks, which are usually performed using different procedures from control theory, as if they were applied to smooth dynamical systems. For instance, averaging, describing function and Hamel locus methods have been applied, possibly leading to inaccurate predictions about the real dynamics of the non-smooth system. Moreover, the analysis of the dynamical behavior of these systems is conventionally performed case by case, clearly distinguishing between parallel and series resonant inverters.

In this paper, first, a unified circuit diagram and its equivalent non smooth model have been derived allowing to perform a unified and rigorous mathematical study for both systems through the introduction of a continuous parameter (called $\beta$ in this paper), which also takes into account the parasitic elements. The extreme cases $\beta=0$ and $\beta=1$ correspond to the ideal series and to the ideal parallel implementations respectively. Using this generalized model, non smooth bifurcation phenomena in LC resonant parallel and series inverters under zero current switching strategy are studied. Analytical expressions, for both the limit sets and the bifurcation values of the parameters, have been found, thus providing more understanding and quantitative information for real circuits than in previous literature. The bifurcation scheme explains the coexistence of different attractors in these inverters, giving details in both the state plane and the parameter plane to prevent undesired dynamics. Hence, the model and the methods presented in this work can help to design LC resonant inverters more effectively, which is especially challenging if a global picture of all the possible dynamics is of concern. Some issues regarding applications like amplitude and frequency of oscillations, that are highly useful in control design, are also determined while avoiding, whenever it was possible, numerical procedures.

\section{References}

1. Suryawanshi, H.M., Tarnekar, S.G.: Resonant converter in high power factor, high voltage DC applications. IEE Proc. - Electr. Power Appl. 145, 4, 307-314 (1998)

2. Schwarz, F.C.: An Improved Method of Resonant Current Pulse Modulation for Power Converters. IEEE Trans. on Ind. Electron. and Control Instrum. 23, 2, 133-141 (1976)

3. Steigerwald, R.L., Ngo, K.D.T.: Full bridge lossless switching converter. U.S. Patent 4864479 (1989)

4. Samanta, S., Rathore, A.K.: A New Current-Fed CLC Transmitter and LC Receiver Topology for Inductive Wireless Power Trans- fer Application: Analysis, Design, and Experimental Results. IEEE Trans. on Transportation Electrification. 1, 4, 357-368 (2015)

5. Fernandes, R.C, de Oliveira, A.A.: Theoretical bifurcation boundaries for Wireless Power Transfer converters. IEEE 13th Brazilian Power Electron. Conf. and 1st Southern Power Electron. Conf. (COBEP/SPEC). 1-4., Fortaleza (2015)

6. Bonache-Samaniego, R., Olalla, C., Martínez-Salamero, L., Maksimovic, D.: $6.78 \mathrm{MHz}$ self-oscillating parallel resonant converter based on GaN technology. 2017 IEEE Applied Power Electron. Conf. and Expos. (APEC). 1594-1599, Tampa, FL (2017)

7. Deng, J., Li, S., Hu, S., Mi, C.C., Ma, R.: Design Methodology of LLC Resonant Converters for Electric Vehicle Battery Chargers. IEEE Trans. on Veh. Technol. 63, 4, 1581-1592 (2014)

8. Baya, B.: Simplified model of resonant inverters for the modelisation of induction heating of billet. IECON 40th Annual Conference of the IEEE Ind. Electron. Soc. 3270-3276, Dallas (2014)

9. Schnell, R.W., Zane, R.A., Azcondo, F.J.: HID lamp driver with phase controlled resonant-mode ignition detection and fast transition to LFSW warm-up mode. IEEE 12th Workshop on Control and Model. for Power Electron. (COMPEL). 1-8, Boulder, CO (2010)

10. Erickson, R.W., Maksimovic, D.: Fundamentals of Power Electronics. Lluwer, Springuer (2001)

11. Bonache-Samaniego, R., Olalla, C., Martínez-Salamero, L.: Design of self-oscillating resonant converters based on a variable structure systems approach. IET Power Electron. 57, 1, 111-119 (2015)

12. Ehsani, M.: Power conversion using zero current soft switching. U.S. Patent 5287261 (1994)

13. Andronov, A.A., Vitt, A., Khaikin, S.E.: Theory of oscillators. Pergamon Press, Oxford (1966)

14. di Bernardo, M., Budd, C.J., Champneys, A.R., Kowalczyk, P.: Piecewise-Smooth Dynamical Systems: Theory and Applications, Appl. Math. Sci. 163, Springer-Verlag London Ltd., London (2008)

15. Filippov, A.F., Arscott, F.M.: Differential Equations with Discontinuous Right-Hand Sides. Kluwer Academic, Dordrecht (1988).

16. Kuznetsov, Yu.A., Rinaldi, S., Gragnani, A.: One-Parameter Bifurcations in Planar Filippov Systems. Int. J. Bifurc. Chaos 13, 2157-2188 (2003)

17. Freire, E., Ponce, E., Torres, F.: Canonical discontinuous planar piecewise linear systems. SIAM J. Appl. Dyn. Syst. 20, 11, 181211 (2012)

18. Freire, E., Ponce, E., Torres, F.: A general mechanism to generate three limit cycles in planar Filippov systems with two zones. Nonlinear Dynamics 78, 251-263 (2014)

19. Freire, E., Ponce, E., Torres, F.: On the critical crossing cycle bifurcation in planar Filippov systems. J. Differ. Eq. 259, 7086-7107 (2015)

20. Coll, B., Gasull, A., Prohens, R.: Degenerated Hopf bifurcation in discontinuous planar systems. J. Math. Anal. Appl. 253, 671-690 (2001)

21. Han, M., Zhang, W.: On Hopf bifurcation in non-smooth planar systems. J. Differ. Eq. 248, 2399-2416 (2010)

22. Chen, X., Romanovski, V., Zhang, W.: Degenerate Hopf bifurcations in a family of FF-type switching systems J. Math. Anal. Appl. 432, 1058-1076 (2015)

23. Chen, X., Llibre, J., Zhang, W.: Averaging approach to cyclicity of Hopf bifurcations in planar linear-quadratic polynomials discontinuous differential systems. Disc. Cont. Dyn. Syst., 22, 10, 3953-3965 (2017)

24. Giannakopoulos, F., Pliete, K.: Planar systems of piecewise linear differential equations with a line of discontinuity. Nonlinearity 14 , 1611-1632 (2001) 